\title{
Differentiation of Exophytic Renal Masses with Determination of the Angular Interface with Renal Parenchyma in US and $\mathrm{CT}^{*}$
}

\author{
Duzgun Yildirim $^{1}$, Halil Bozkurt ${ }^{2}$, Abdullah Cirakoglu ${ }^{3}$, Mutlu Sahin $^{4}$, Bengi Gurses ${ }^{5}$, Baki Ekci $^{6}$ \\ ${ }^{1}$ Department of Radiology, Iskenderun Military Hospital, Hatay, Turkey \\ ${ }^{2}$ Department of Urology, Karaman Public Hospital, Karaman, Turkey \\ ${ }^{3}$ Department of Urology, Kasimpasa Military Hospital, Istanbul, Turkey \\ ${ }^{4}$ Department of Surgery, University of Washington, Seattle, USA \\ ${ }^{5}$ Department of Radiology, Yeditepe Unversity Hospital, Istanbul, Turkey \\ ${ }^{6}$ Department of Surgery, Yeditepe Unversity Hospital, Istanbul, Turkey \\ Email: yildirimduzgun@yahoo.com
}

Received April 17, 2012; revised May 7, 2012; accepted May 19, 2012

\begin{abstract}
Purpose: To determine whether benign exophytic renal masses can be distinguished from malignant lesions by using the angular interface sign in ultrasonography (US) and computerized tomography (CT). Materials and Methods: A total of 71 cases with exophytic renal mass $(2 \mathrm{~cm}$ or greater) were examined on the basis of angular interface in US $(\mathrm{n}=$ 23), CT $(n=21)$ and US + CT $(n=16)$ between January 2008 and June 2010 were included in this study. The renal interface relationships were examined by 2 radiologists and classified as having angular or wide interface. Results: No statistically significant difference was found between the findings of two readers. There was almost perfect interobserver agreement for the interface sign. For cystic lesions, the angular interface sign was determined in all but two Bosniak category 1 case. Also, the angular interface sign was positive in all but one Bosniak category 2 - 3. For cystic lesions with solid component and pure solid lesions, in the benign group, the angular interface sign was positive in all except three cases (vascular malformation, oncocytoma and Xanthogranulomatous pyelonephritis). In the malignant group, the angular interface sign was determined in only two RCC cases; in other primary or metastatic malignant lesions there was a wide interface sign. Conclusion: Exophytic renal masses can be differentiated as malignant or benign with $87 \%$ accuracy using only the angular interface sign in US or CT and also in opposition to dynamic-contrast examinations. This method entails a lack of additional radiation or contrast media exposure, time-saving, and costeffectivity.
\end{abstract}

Keywords: Renal Neoplasms; Differential Diagnosis; Ultrasonography; Computed Tomography; Sign

\section{Introduction}

Until now, many studies have been conducted using US, CT or MRI to evaluate renal masses [1-4]. In these studies differential diagnosis was attempted with various, complex modalities like contrast enhanced US, dynamic or perfussion CT/MRI, and diffusion MRI. Despite these detailed, difficult and often expensive modalities, the evaluation of renal masses remains a common problem in radiologic practice [4]. The Bosniak classification of renal cysts has been used widely for interpretation of charecteristics of cystic renal masses. Although the known sensitivity and specificity values, and of time consumption, contrast enhanced modalities for further

${ }^{*}$ Conflict of interest disclosure: The authors declared no conflicts of interest. evaluation of Bosniak 3 or higher category, a biopsy is also needed. For this reason an alternative sign is needed other than complex algorithms which should be practical, easy and useful both in US and CT examinations [5]. Having inspired from a previous study which performed with the guidance of MRI ([6], Verma et al.), we have noted that some masses have a wide-rounded interface with the renal parenchyma, whereas others have a pyramidal-triangular interface, with a definable apex within the parenchyma. We used also the same term for CT and also for US.

In this retrospective study, our aim is to test the effectiveness of the renal angular interface sign in exophytic renal masses to differentiate benign and malignant lesions in US and CT that is studied with MRI before (sensitivity $78 \%$, specifity $100 \%$ ) by Verma et al. [6]. 


\section{Materials and Methods}

\subsection{Patients}

All the patients whose were recorded as having renal mass(es) in our image archieve, between January 2008 and June 2010 were studied retrospectively. Cases with a renal mass equal and/or greater than $2 \mathrm{~cm}$ in longer axis $(n=86)$ studied furtherly. But, four $(n=4)$ cases that had innumerable lesions which were too close to each other were excluded from the study (2 polycystic kidney, 1 multiple angiomyolipoma, 1 RCC) because of the possible deforming effect on the neighboring lesion's interface sign. Besides lesions having a diameter less than $2 \mathrm{~cm}$, non-exophytic lesions originating from atrophic renal parenchyma, cases where the final diagnosis was not achieved were also excluded from the study $(n=11)$.

A total of 71 cases with exophytic renal mass $(2 \mathrm{~cm}$ or greater) examined prospectively on the basis of angular interface in US $(n=23)$, CT $(n=21)$ and US + CT $(n=$ 16) appearences between January 2008 and June 2010 were included in this study. Of 71 exophytic renal mass cases, 56 had single lesions and 15 had multiple lesions (7 benign cases with 4 polycystic renal syndrome, 2 multiple angiomyolipoma, 1 multifocal oncocytoma and 8 malignant cases with 2 pulmonary metastasis, 2 RCC, 2 AML, 2 lymphoma metastasis). In multiple lesions the dominant one (the biggest or most septated, enhanced or having more solid component lesion) was examined. For all lesions, we had both related horizontal or coronal image copies. In all included cases (women: 27; men: 44; mean age: 47), the diameter of renal masses was $2 \mathrm{~cm}$ or greater (mean: $43 \mathrm{~mm}$ ). The diagnosis of the cases included in this study was confirmed via MRI $(n=23)$, typical radiological images $(n=13)$, follow-up data $(n=$ $11)$ and biopsy $(n=24)$.

\subsection{Technique and Image Analysis}

CT imaging was performed with 16 dedector multislice CT system (Somatom Sensation 16-slice CT). With processing the recorded row data, axial and reformatted coronal images were produced. US images which achieved by $7.5 \mathrm{mHz}$ convex transducer (Ultrason için: GE Logic 9 Ultrasound Imager-Milwaukee, WI-) were also scrutinized to obtain the proper coronal and axial image planes thorough the targetted renal exophytic lesion. Exophytic masses of included cases classified as having wide interface (which have rounded interface with the renal parenchyma) or angular interface (which have pyramidal-triangular renal interface) regarding the masskidney parenchyma relations. If the angular interface was detected in any of the examined coronal or horizontal images, the sign was accepted as positive.

\subsection{Statistical Analysis}

The interface sign was evaluated by two independent readers. Neither of these two readers was aware of the diagnostic data. Only the digital images were evaluated as to whether they had the angular interface sign or not. Interobserver agreement was evaluated by Kappa analysis of the dataset which was transferred to an SPSS program. All the obtained data analysed statistically in $2 * 2$ tables and sensitivity, specifity, accuracy, positive and negative predictive values were calculated in differentiation of malignant and benign exophytic renal masses.

\section{Results}

For cystic lesions, the angular interface sign was determined in all but two Bosniak category 1 cases. The angular interface sign was also positive in all but one Bosniak category 2 - 3 .

For cystic lesions concerning solid components and pure solid lesions, in the benign group the angular interface sign was positive in all except three, that is, 1 vascular malformation, 1 oncocytoma and 1 Xanthogranulomatous pyelonephritis. In the malignant group, the angular interface sign was determined only in two RCC cases. With other primary or metastatic malignant lesions there was a wide interface sign. Renal interface sign characteristics are demonstrated in Figures 1-3.

There was almost perfect interobserver agreement for interface signs (Kappa: 0.97). We thought this so high degree of agreement is dependent to the simplicity of the procedure and method that has been used. According to these data (the mean values from the two readers); of all benign cases $(n=48)$, in 6 patients, the angular interface sign has not been determined (evaluated as false negative). On the other hand, in malignant cases $(n=23)$, the sign was also positive (evaluated as false positive) in 2 patients. With the evaluation of all the obtained data statistically, malignant versus benign differentiation could

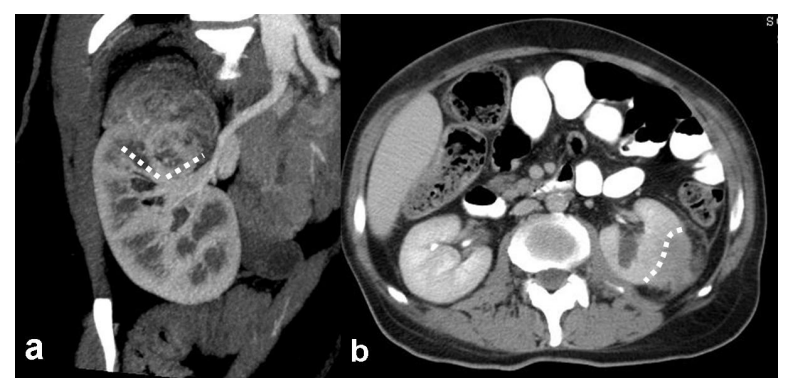

Figure 1. Investigated findings of the interface relationship for three different cases which are variated lesions of the right kidney zone. (a) Simple exophytic cortical cyst, triangular interface; (b) Primary marginal zone lymphoma case of renal focus, wide interface; (c) Multiseptate, heterogeneous echoe cystic lesions; triangular interfaces at dominant cystic mass (dashed lines). 


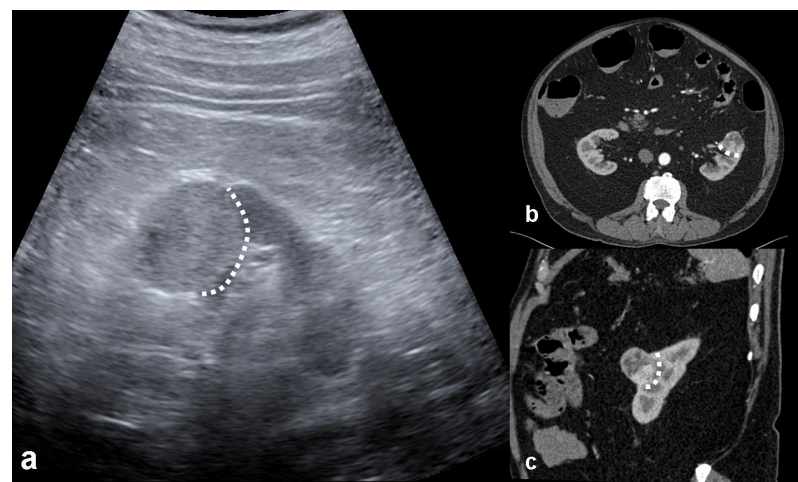

Figure 2. (a) Atypical angiomyolipoma; heterogeneous density lesion arising from the upper pole of the right kidney, coronal reformatted contrast-enhanced multidetector CT image. Triangular interface; (b) A case of papillary type RCC; axial view in the contrast-enhanced CT section, large interface (dashed lines).
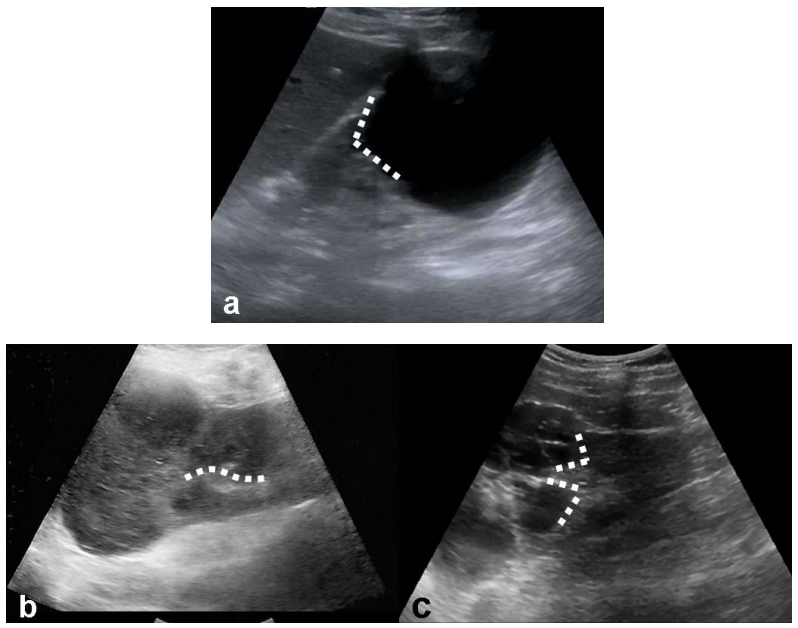

Figure 3. The reported cases that excisional biopsy compatible with incidental RCC of US appearance monitored as: (a) Echogenic; (b) Axial; and (c) Coronal, noteworthy heterogeneous enhancement on contrast-enhanced CT images. Renal interface relationship of the lesion is observed widely (dashed lines).

be achieved with $87.5 \%$ sensitivity, $91 \%$ specifity and $87 \%$ accuracy values. Specifically, the accuracy value for differentiation in complicated cystic lesions was $85 \%$ whereas it was only $65 \%$ for solid ones.

\section{Discussion}

In differential diagnosis of renal masses, many different modalities like US, CT, MRI, PET-CT have been used up to now, and many studies were designed using different protocols upon these modalities [1-4]. In these studies using contrast US, dynamic-diffusion-perfusion software which means functional, cross-sectional examinations, the characterization and classification of renal masses were attempted [2,5]. Despite these complex, difficult and often expensive modalities, it is not possible to diagnose renal masses with $100 \%$ accuracy. The diagnosis of non-contrast enhancing renal masses of any size is difficult. To differentiate benign and malign lesions in these types of masses, advanced examinations using many parameters are needed [5]. After all of these difficult studies a differential feature is actually not presented very clearly, but only some clues have been obtained.

We applied a new method on US and CT which had been used by Verma et al. on MRIs previously. Our aim in this study was to determine the availability level of discrimination that only could be reached with the help of more complicated modalities, and if it might be valid for US and CT establishments. Although previous methods and studies show that the diagnostic performance can be affected by interobserver variations especially in differentiation of renal lesions [7], interobserver agreement was absolute (exact) in our study. Concerning the other methods; during the diagnosis of renal lesions, even pure cystic components or some of the lipomatous content lesions predicted as angiomyolipoma might result in RCC after surgery. As a result, the reliable parameters of benignity emerged into a controversial situation [8,9].

As an alternative to evaluating the imaging and contrast characteristics is to follow the growth rate of the lesion. It is reported that a malign mass grows more than mean $0.3-0.5 \mathrm{~cm} /$ year. Despite the usage of electronic parameters in measurement, it is still subjective and there are benign lesions growing as well as malignant lesions grow slowly. This is not a suitable diagnostic choice for suspicious renal masses because of the possible radiation exposure and high cost resulting from frequent CT controls [10]. Additionally, the growth of masses in follow-up is an undesirable condition for treatment because $<4 \mathrm{~cm}$ masses can be treated by laparoscopic methods with low morbidity and excellent tumoral control. Regarding the complexity of these tests and algorhytms, Verma et al. reported that the renal interface sign at MRI is helpful in differential diagnosis of exophytic renal masses [6].

In this retrospective study we aimed to search the existence of the same sign in US and CT that are used at least as MRIs in the evaluation of renal masses. As in this study, we present a simple, practicle morphologic method for assessing exophytic renal mass in differentiaition of benign versus malignant lesions. Easy applicability and repeatability of this sectional method is not about lesion dynamics but only defines the shape of the interface of lesion with the renal parenchyma. Although previously defined with MRI; in incompatible cases, MRI-contrendicatied cases or in fast-emergency conditions, the sign can be achieved by CT tor US. With high interobserver agreement value, our results suggest that if an exophytic renal mass is difficult to differentiate on the 
basis of other imaging characteristics alone, determining the lesion-kidney interface type with tomographic (on CT) or sonographic (on US) examination can assess the lesion nature with high accuracies (as high as 87\%).

The major limitation for our study is the low number of renal mass cases that prevents making cystic-solid lesion groups and assessing the better distinctive characteristics of this sign for both groups. Further studies with a higher number of cases are needed to test the effectiveness of this sign, especially in Bosniak 3 lesions when it is hard to make a decision about follow-up or surgery. The major limitation for this method is that the mass should be $2 \mathrm{~cm}$ or greater and must be of an exophytic nature. Renal masses are usually detected incidentally at larger sizes, and the neoplasia originating from renal collecting system is not very frequent. For this reason we do not think that these limitations are so significant. On the other hand, because the iodine based contrast material causes anaphylaxis risk and MRI contrast causes nephrogenic systemic sclerosis risk, the usage of this angular interface sign in non-contrast examinations for exophytic renal masses may be invaluable [11]. The same concept is also valid for renal insufficiency because of contrast material contraindication.

In conclusion, exophytic renal masses can be differentiated as malignant or benign with high specificity (91\%) and accuracy (87\%) using only the angular interface sign in also US or CT.

\section{REFERENCES}

[1] J. B. Zhang, R. A. Lefkowitz, N. M. Ishill, L. Wang, C. S. Moskowitz, P. Russo, H. Eisenberg and H. Hricak, "Solid Renal Cortical Tumors: Differentiation with CT," Radiology, Vol. 244, No. 2, 2007, pp. 494-504. doi:10.1148/radiol.2442060927

[2] G. M. Israel, N. Hindman and M. A. Bosniak, "Evaluation of Cystic Renal Masses: Comparison of CT and MR Imaging by Using the Bosniak Classification System," Radiology, Vol. 231, No. 2, 2004, pp. 365-371. doi:10.1148/radiol.2312031025
[3] O. Hélénon, J. Correas, C. Balleyguier, M. Ghouadni and F. Cornud, "Ultrasound of Renal Tumors," European Radiology, Vol. 11, No. 10, 2001, pp. 1890-1901. doi:10.1007/s003300101077

[4] S. R. Prasad, N. C. Dalrymple and V. R. Surabhi, "CrossSectional Imaging Evaluation of Renal Masses," Radiologic Clinics of North American, Vol. 46, No. 1, 2008, pp. 95-111. doi:10.1016/j.rcl.2008.01.008

[5] G. M. Israel and M. A. Bosniak, "MR Imaging of Cystic Renal Masses," Magnetic Resonance Imaging Clinics of North America, Vol. 12, No. 3, 2004, pp. 403-412. doi:10.1016/j.mric.2004.03.006

[6] S. K. Verma, D. G. Mitchell, R. Yang, C. G. Roth, P. O’Kane, M. Verma and L. Parker, "Exophytic Renal Masses: Angular Interface with Renal Parenchyma for Distinguishing Benign from Malignant Lesions at MR Imaging,” Radiology, Vol. 255, No. 2, 2010, pp. 501-507. doi:10.1148/radiol.09091109

[7] C. L. Siegel, E. G. McFarland, J. A. Brink, A. J. Fisher, P. Humphrey and J. P. Heiken, "CT of Cystic Renal Masses: Analysis of Diagnostic Performance and Interobserver Variation,” American Journal of Roentgenology, Vol. 169, No. 3, 1997, pp. 813-818.

[8] E. K. Outwater, M. Bhatia, E. S. Siegelman, M. A. Burke and D. G. Mitchell, "Lipid in Renal Clear Cell Carcinoma: Detection on Opposed-Phase Gradient-Echo MR Images," Radiology, Vol. 205, No. 1, 1997, pp. 103-107.

[9] J. K. Kim, T. K. Kim, H. J. Ahn, C. S. Kim, K. R. Kim and K. S. Cho, "Differentiation of Subtypes of Renal Cell Carcinoma on Helical CT Scans," American Journal of Roentgenology, Vol. 178, No. 6, 2002, pp. 1499-1506.

[10] W. Siu, K. S. Hafez, W. K. Johnston III and J. S. Wolf Jr., "Growth Rates of Renal Cell Carcinoma and Oncocytoma under Surveillance are Similar,” Urologic Oncology, Vol. 25, No. 2, 2007, pp. 115-119. doi:10.1016/j.urolonc.2006.07.018

[11] S. K. Morcos, H. S. Thomsen, J. A. W. Webb and Members of the Contrast Media Safety Committee of the European Society of Urogenital Radiology (ESUR), "Contrast-Media-Induced Nephrotoxicity: A Consensus Report,” European Radiology, Vol. 9, No. 8, 1999, pp. 1602-1613. doi:10.1007/s003300050894 